\title{
Bound-state formation on a spherical shell: A model for superconductivity of alkali-metal-doped $\mathbf{C}_{60}$
}

\author{
Z. Gedik and S. Ciraci \\ Department of Physics, Bilkent University, Bilkent 06533, Ankara, Turkey
}

(Received 2 July 1991)

\begin{abstract}
We show that an attractive interaction between two electrons confined to the surface of a sphere gives rise to a bound state, no matter how weak the interaction is. We explore the similarity between a sphere and a (two-dimensional) plane as far as pairing properties are concerned. We also discuss the relevance of the model to a recently discovered superconductor, alkali-metal-doped $\mathrm{C}_{60}$.
\end{abstract}

The cage-structure carbon clusters, $\mathrm{C}_{m}$, have been candidates for unusual materials providing novel properties. ' It is now contemplated that $\mathrm{C}_{m}$, a superatomlike basis, can form crystals with adjustable properties since the size and the symmetry are controlled by $m$. Recently, $\mathrm{C}_{60}$ has been shown to be stable in the truncated-icosahedron structure. $^{2}$ Moreover, $\mathrm{C}_{60}$ was found to be a direct-bandgap semiconductor in the solid phase. ${ }^{3}$ Self-consistent field (SCF) calculations based on the local-density approximation predict that this solid phase is stable in the fcc structure with a $1.6 \mathrm{eV}$ (per basis) cohesive energy and has a direct band gap of $1.5 \mathrm{eV}$. The calculated electronic structure of the solid $\mathrm{C}_{60}$ indicates that intermolecular interactions are weak due to the small overlap of molecular orbitals. ${ }^{3,4}$ Nearest-neighbor interaction in the solid phase can be compared with the interlayer interaction in graphite. The latter is known to be weak. In fact, photoemission measurements ${ }^{5}$ along with the results of those SCF calculations imply that the electronic states of the solid phase can be described to some extent by the states of isolated $\mathrm{C}_{60}$. On the other hand, the $\mathrm{C}_{60}$ structure can be visualized as a single, two-dimensional (2D) graphite layer consisting of pentagons and hexagons which is wrapped on a sphere. The effective dimensionality of this sphere and properties in conjunction with it are already of interest for studies on low-dimensional electron systems.

More recently, $\mathrm{C}_{60}$ has been found to be a superconductor after treatment with alkali-metal atoms. The apparent $T_{c}$ of $\mathrm{K}_{x} \mathrm{C}_{60}, \mathrm{Rb}_{x} \mathrm{C}_{60}$, and $\mathrm{Cs}_{x} \mathrm{C}_{60}(x \simeq 3)$ samples were measured at 18,28 , and $30 \mathrm{~K}$, respectively. ${ }^{6-9}$ In the isolated $\mathrm{C}_{60}$, the lowest unoccupied molecular orbital (LUMO) state is $\simeq 5 \mathrm{eV}$ below vacuum level and also is $1.9 \mathrm{eV}$ above the highest occupied molecular orbital (HOMO) state. $^{3-5}$ The valence electron of an alkalimetal atom has low affinity, and thus can easily be donated to LUMO. A similar situation was already pointed out for $\mathrm{K}$ and $\mathrm{Na}$ adsorbed on Si surfaces. ${ }^{10}$ According to the results of the SCF pseudopotential calculations based on the local-density approximation, the alkali-metal atoms are adsorbed at the centers of the hexagonal rings above the atomic plane. These are the low-charge-density locations on the surface. At low coverage, the adsorbed alkali atoms donate their valence electrons to the empty surface states which attributes a 2D metallic character to the semiconductor surface. It is now interesting to understand how the alkali-metal valence electrons occupying the LUMO state are paired in a fullerene, and how a pair can move in its ordered phase (fullerite).

In this paper we investigate the two-electron problem on a sphere representing a single $\mathrm{C}_{60}$ molecule with electrons donated from adsorbed alkali atoms. We found that two electrons on a sphere form a bound state no matter how weak the attractive interaction is. This suggests that the superconductive phase of alkali-metal-doped $\mathrm{C}_{60}$ solid is achieved upon the formation of electron pairs on fullerenes. These pairs can move between adjacent fullerenes via Josephson-like tunneling. The validity of the model depends upon to what extent a $\mathrm{C}_{60}$ molecule has spherical symmetry and whether the intermolecular overlap integrals are small enough to preserve the localized nature of LUMO electrons in the solid phase.

We first consider two particles moving on the surface of a sphere which are interacting with each other via a potential depending upon relative coordinates of the particles only. Since this problem can be reduced to an effective one-body problem, or central force problem on the surface of a sphere, it is enough to study the motion of a particle in the field of an attractive potential. As is well known, in one- and two-dimensional cases, a bound state is formed no matter how weak the attractive interaction is. "In $3 \mathrm{D}$, on the other hand, one requires a critical coupling strength to form a discrete level below the continuous spectrum. Note that this is not in contradiction with the Cooper problem where two electrons are always bound since they are above a Fermi surface of a many-electron system. In our system, there are only a few free electrons on a $\mathrm{C}_{60}$ sphere, so a Fermi surface cannot be defined.

In spite of the fact that the solid $\mathrm{C}_{60}$ is a $3 \mathrm{D}$ system, the surface of a $\mathrm{C}_{60}$ molecule has $2 \mathrm{D}$ character. Consequently, one expects behavior reminiscent of the perfect 2D case, i.e., a bound state even for a very weak attractive interaction. To verify this conjecture, we first evaluate the Green's function $G_{0}$ for the free particle, and then treat the attractive interaction perturbatively. We sum the infinite perturbation series to find the Green's function $G$ and show that it has a pole at negative energies.

The Green's function for a free particle of mass $m$ con- 
strained to move on the surface of a sphere of radius $R$ is given by

$$
G_{0}\left(\hat{\mathbf{n}}, \hat{\mathbf{n}}^{\prime} ; \varepsilon\right)=\frac{1}{4 \pi E_{R}} \sum_{l=0}^{\infty} \frac{2 l+1}{\varepsilon-l(l+1)} P_{l}\left(\hat{\mathbf{n}} \cdot \hat{\mathbf{n}}^{\prime}\right)
$$

where $\hat{\mathbf{n}}$ and $\hat{\mathbf{n}}^{\prime}$ are the position vectors on the unit sphere, $P_{l}$ is a Legendre polynomial, and $\varepsilon$ is the energy of the particle in units of $E_{R}=\hbar^{2} / 2 m R^{2}$. Equation (1) can be verified easily by using the fact that eigenstates are given by spherical harmonics $Y_{l}^{m}(\theta, \phi)$.

We now assume that a weak attractive interaction $V_{0}$ is effective in a solid angle $\boldsymbol{\Omega}_{0}$. Under these circumstances we examine the Green's function $G\left(\hat{\mathbf{n}}, \hat{\mathbf{n}}^{\prime} ; \varepsilon\right)$ to find if it has a pole in the interval $\left[-V_{0}, 0\right]$. Since $V_{0} \rightarrow 0^{+}$for a weak interaction, we have to find $G\left(\hat{\mathbf{n}}, \hat{\mathbf{n}}^{\prime} ; \varepsilon\right)$ as $\varepsilon \rightarrow 0^{-}$. In this limit $G_{0}\left(\hat{\mathbf{n}}, \hat{\mathbf{n}}^{\prime} ; \varepsilon\right)$ can be evaluated in a closed form. For this purpose, we approximate the summation in Eq. (1) by neglecting $\varepsilon$ dependence of all the terms except $l=0$. Noting that $(2 l+1) / l(l+1)=1 / l+1 /(l+1)$, we obtain ${ }^{12}$

$$
G_{0}\left(\hat{\mathbf{n}}, \hat{\mathbf{n}}^{\prime} ; \varepsilon\right) \simeq \frac{1}{4 \pi E_{R}}\left(\frac{1}{\varepsilon}+\ln \frac{1-\hat{\mathbf{n}} \cdot \hat{\mathbf{n}}^{\prime}}{2}+1\right)
$$

Here, the logarithm term indicates that the above model is similar to the 2D free particle problem. It is seen that $G_{0}$ exhibits a logarithmic singularity as the two points approach each other. This is consistent with the observation that a very small portion of the surface of a sphere can be approximated by a $2 \mathrm{D}$ plane. The singularity of $G_{0}$ and $\varepsilon$ goes to zero in Eq. (2) is stronger than $G_{0}$ of the $2 \mathrm{D}$ plane which changes with the logarithm of $\varepsilon$. "Such a difference is expected since the energy spectra are quite different in the two cases. In fact, $1 / \varepsilon$ behavior in Eq. (2) instead of $\ln \varepsilon$ as in the perfect 2D case originates from discretization of the energy levels. If the difference between these discrete energy levels becomes very small, then the approximation made to obtain Eq. (2) is no longer valid. In this case, we cannot separate out $1 / \varepsilon$ and neglect the $\varepsilon$ dependence of the other terms, but consider all the terms of the form $1 /(\varepsilon-x)$ where $x$ is now a continuous variable instead of discrete $l(l+1)$. Adding those terms by integration over $x$, we end up with a logarithmic singularity. This is an expected result since the spacing of discrete energy levels is controlled by $R$, and the sphere approaches a plane as $R$ increases indefinitely. It is seen that the Green's functions for a particle on the surface of a sphere and on the (2D) plane are similar in the appropriate limits as far as the position $\left(\hat{\mathbf{n}}, \hat{\mathbf{n}}^{\prime}\right)$ and energy $(\varepsilon)$ dependences are concerned.

Knowing $G_{0}$ and $V_{0}$, we can find by means of the perturbation expansion

$$
\begin{array}{rl}
G\left(\hat{\mathbf{n}}, \hat{\mathbf{n}}^{\prime} ; \varepsilon\right)=G_{0}\left(\hat{\mathbf{n}}, \hat{\mathbf{n}}^{\prime} ; \varepsilon\right)-V_{0} \int_{\Omega_{0}} & d \hat{\mathbf{n}}_{1} G_{0}\left(\hat{\mathbf{n}}, \hat{\mathbf{n}}_{1} ; \varepsilon\right) \\
& \times G_{0}\left(\hat{\mathbf{n}}_{1}, \hat{\mathbf{n}}^{\prime} ; \varepsilon\right)+\cdots .
\end{array}
$$

Here, for the sake of simplicity we assume that $V_{0}$ is a constant interaction, i.e., independent of the relative positions of the electrons, and is effective only in the solid angle $\Omega_{0}$. For $\hat{\mathbf{n}} \cdot \hat{\mathbf{n}}^{\prime} \neq 1$ and $\varepsilon \neq 0, G_{0}$ is finite. Thus, $G$ can be calculated by summing the series. We approximate the product of the Green's functions by factoring out $G_{0}$ and by using the average values $\left\langle G_{0}\right\rangle_{\Omega_{0}}$ for the rest. Therefore, we calculate $\left\langle G_{0}\right\rangle_{\Omega_{0}}$, which is found to be $\left[1 / \varepsilon+4 \delta^{2}\left(\frac{1}{2} \ln \delta-\frac{1}{4}\right)+1\right] / 4 \pi E_{R}$ where $0<\delta<1$. Since $\varepsilon \rightarrow 0^{+},\left\langle G_{0}\right\rangle_{\Omega_{0}}$ can be very well approximated by $1 / 4 \pi E_{R} \varepsilon$. At the end we obtain

$$
G\left(\hat{\mathbf{n}}, \hat{\mathbf{n}}^{\prime} ; \varepsilon\right)=\frac{G_{0}\left(\hat{\mathbf{n}}, \hat{\mathbf{n}}^{\prime} ; \varepsilon\right)}{1+V_{0} \Omega_{0} / 4 \pi E_{R} \varepsilon} .
$$

It is seen that a negative energy level $E=\varepsilon E_{R}$ $=-V_{0} \Omega_{0} / 4 \pi$ corresponding to a bound state is always formed, even for a very weak interaction. The origin of this interaction is beyond the scope of this study. Nevertheless, we assume that the net interaction between the electrons on the sphere is attractive. In principle, $V_{0}$ contains Coulomb repulsion and an attractive mechanism, most probably due to the vibrational modes of fullerene. Note that the wave function of the two-electron system has to by antisymmetrized. However, the energy eigenvalues remain unchanged after the antisymmetrization.

So far we have shown that a two-electron system on a sphere is unstable against pair formation. We next consider a solid phase formed by the spheres in the foregoing discussion. When these spheres are placed at lattice sites they begin to interact with each other weakly. The superconductivity has been observed for approximately three alkali-metal atoms per $\mathrm{C}_{60}$ molecule. Two of these three electrons will fill the first conduction band while the third one creates a half-filled metallic band. Therefore, effectively we are left with one electron per $\mathrm{C}_{60}$ molecule and these electrons are free to move from site to site. Thus, we can assume that two electrons can come together on a sphere to form a bound state as we discussed above. The increase in the Coulomb energy due to the occupation of a sphere by a fourth electron is expected to be negligible because these materials exhibit metallic behavior in the normal phase.

Note that superconductivity is not achieved by BoseEinstein condensation even though the electrons move in the form of tightly bound pairs. Otherwise, the measured critical temperature would require an on-site interaction of 30-40 eV since the value of transfer (hopping) integral inferred from band-structure calculations is only $\sim 0.1$ $\mathrm{eV}$. In view of this argument, we propose that superconductivity occurs as a result of formation of pairs in units (i.e., on $\mathrm{C}_{60}$ spheres), which are coupled by Josephson interaction. The situation is reminiscent of the superconductivity of layered systems where the 2D Fermi liquids in the layers are unstable against Cooper pairing and they interact via interlayer tunneling. In the present case layers are replaced by spheres which can be occupied by only a few electrons and the origin of the pairing is not Cooper instability but a dimensionality effect. For layered materials it can be shown that the critical temperature $T_{c}$ is not altered by the Josephson coupling. ${ }^{13}$ Therefore, in the present case it is expected that $k_{B} T_{c} \sim V_{0} \Omega_{0}$, which leads to a reasonable value for the coupling constant $V_{0}$. Since the infinite layers are replaced by finite spheres, charging effects due to the occupation of a sphere by an excess pair can be important. In fact, treating the $\mathrm{C}_{60}$ molecules as 
spherical capacitors we find that charging energy is a few eV. This implies that the $\mathrm{C}_{60}$ molecule can be occupied by only one pair (formed by the third and the fourth electrons donated by the alkali-metal atoms) when the solid is in the superconductive phase. Nevertheless, for a correct description of the system, a 3D net of Josephson junctions including charging effects should be studied in detail.

In conclusion, we have shown that in analogy to 2D and ID systems, an attractive interaction always yields a bound state for particles constrained to move on the surface of a sphere. In the crystal composed of those spheres, a transition to the superconducting phase associated with the formation of pairs can be observed. Such a mechanism can be thought to be operational in the superconductivity of the alkali-metal-doped $\mathrm{C}_{60}$ solid. Nevertheless, existing data about this class of materials are incomplete, and do not allow us to draw conclusions about the origin of the attractive interaction and the nature of the mechanism of the superconductivity.

This study was partially supported by the joint study agreement between Bilkent University and IBM Zurich Research Laboratory.
'E. A. Rohlfing, D. M. Cox, and A. Kaldor, J. Chem. Phys. 81, 3322 (1984).

${ }^{2}$ W. Krätschmer, L. D. Lamb, K. Fostiropoulos, and D. R. Huffman, Nature (London) 347, 354 (1990).

${ }^{3}$ Susumu Saito and Atsushi Oshiyama, Phys. Rev. Lett. 66, 2637 (1991).

${ }^{4}$ Q.-M. Zhang, Jae-Yel Yi, and J. Bernholc, Phys. Rev. Lett. 66, 2633 (1991).

5J. H. Weaver, J. L. Martins, T. Komeda, Y. Chen, T. R. Ohno, G. H. Kroll, N. Troullier, R. E. Haufler, and R. E. Smalley, Phys. Rev. Lett. 66, 1741 (1991).

${ }^{6}$ A. F. Hebard, M. J. Rosseinsky, R. C. Haddon, D. W. Murphy, S. H. Glarum, T. T. M. Palstra, A. P. Ramirez, and A. R. Kortan, Nature (London) 350, 600 (1991).

${ }^{7}$ K. Holczer, O. Klein, S.-M. Huang, R. B. Kaner, K.-J. Fu, R. L. Whetten, and F. Diederich, Science 252, 1154 (1991).
${ }^{8}$ M. J. Rosseinsky, A. P. Ramirez, S. H. Glarum, D. W. Murphy, R. C. Haddon, A. F. Hebard, T. T. M. Palstra, A. R. Kortan, S. M. Zahurak, and A. V. Makhija, Phys. Rev. Lett. 66, 2830 (1991).

${ }^{9}$ S. P. Kelty, C.-C. Chen, and C. M. Lieber, Nature (London) 352, 223 (1991).

${ }^{10}$ S. Ciraci and I. P. Batra, Phys. Rev. Lett. 56, 877 (1986); Phys. Rev. B 37, 2995 (1988); I. P. Batra, ibid. 39, 3919 (1989).

''E. N. Economou, Green's Functions in Quantum Physics (Springer-Verlag, Berlin, 1979).

${ }^{12}$ I. S. Gradshteyn and I. M. Ryzhik, Table of Integrals, Series and Products (Academic, San Diego, 1980).

${ }^{13}$ T. Schneider, Z. Gedik, and S. Ciraci, Z. Phys. B 83, 313 (1991). 